Nota Científica

\title{
Relações hipsométricas de ecossistemas de mopane Colophospermum mopane em Mabalane, Província de Gaza, Moçambique
}

\author{
Jacob Miguel Bila1 \\ ${ }^{1}$ Instituto de Investigação Agrária de Moçambique, Avenida das FPLM, 269, CP 3658, Maputo, Moçambique.
}

"Autor correspondente:

jacobila@yahoo.com.br

Termos para indexação:

Ajuste de modelos

Altura das árvores

Parcelas permanentes

Index terms:

Adjust models

Heigh of a tree

Permanent plot
Resumo - Este trabalho teve como objectivo comparar o ajuste dos modelos hipsométricos de Trorey, Henricksen, Stoffels e Curtis nos ecossistemas de mopane (Colophospermum mopane), com base nos dados de parcelas permanentes estabelecidas em Mabalane, Província de Gaza-Moçambique. Foram determinadas as estatísticas de ajustes, coeficiente de determinação $\left(\mathrm{R}^{2 \%} \%\right)$ e erro padrão da estimativa $\left(\mathrm{S}_{\mathrm{yx}} \%\right)$. Fez-se validação do ajustes das equações através do teste Qui-quadrado e análise gráfica de resíduos, ambas satisfatórias para todos os modelos. Os resultados mostraram que o modelo de Stofels teve o melhor ajuste $\operatorname{com}\left(\mathrm{R}^{2}{ }_{\mathrm{aj}} \%\right)$ de 49,92 e erro padrão da estimativa $\left(\mathrm{S}_{\mathrm{yx}} \%\right)$ de 17,97 .

\section{Hypsometric relations of Mopani ecosystems Colophospermum mopane in Mabalane, Gaza Province, Mozambique}

\begin{abstract}
Abstrat - This study aimed to adjust the hypsometric model of Trorey, Henricksen, Curtis and Stoffels in ecosystems of mopane (Colophospermum mopane) based on data from permanent plots established in Mabalane, Gaza Province, Mozambique. Statistical adjustment as coefficient of determination $\mathrm{R}^{2 \%}$ and standard error of estimate $\left(\mathrm{S}_{\mathrm{yx}} \%\right)$ were determined. Adjustment equations were validated using Chi-square and residual graphic analysis made. The results showed that the Stoffels model was presented the best adjustment compared to the other models, with $\mathrm{R}^{2 \%}=49.92$ and $\mathrm{S}_{\mathrm{yx}} \%=17.97$ satisfactory for all tested models.
\end{abstract}

A altura de uma árvore é uma medida dendrométrica importante para a determinação do volume da árvore e consequentemente para a determinação do volume do povoamento florestal. A altura da árvore é medida diretamente no campo, recorrendo-se à sua estimação indireta pela utilização de uma relação hipsométrica. Esta operação permite reduzir o esforço nos trabalhos de inventários florestais, limitando-se a medir a altura de algumas árvores da unidade amostral e estimando-se as demais com base nas equações hipsométricas.

As relações hipsométricas podem ser classificadas em locais ou gerais, dependendo do seu domínio de aplicabilidade. As locais são desenvolvidas em função apenas do diâmetro a 1,30 m e são indicadas para aplicação no povoamento onde se procedeu a coleita dos dados, ou em povoamentos que apresentem características análogas. As relações hipsométricas gerais exprimem a altura da árvore em função do seu diâmetro a 1,30 m, mas recorrem também às variáveis do povoamento, tais como a altura dominante, o diâmetro dominante, a densidade, a idade e, eventualmente, a própria localização do povoamento (Tomé \& Ribeiro, 2007). Porém, o uso indiscriminado desta relação pode levar a erros consideráveis, uma vez que vários fatores 
podem influenciá-la tais como a posição sociológia, índice de sítio, idade, densidade e práticas silviculturais (Barros et al., 2002).

Vários pesquisadores tem estudado a relação entre o diâmetro e a altura das árvores. Veiga et al. (1974) desenvolveram relações hipsómetricas em povoamentos de Eucalyptus sp. onde concluiram que as equações não logarítmicas apresentam maior precisão em relação às equações logarítmicas. Verificaram ainda que não é vantajoso o uso de um único modelo matemático, tanto para a determinação de equações hipsométricas para a altura total, como para as correspondentes às alturas comerciais aos diâmetros de desponta de $8 \mathrm{~cm}$ e $5 \mathrm{~cm}$, pois resultados melhores podem ser obtidos com modelos diferentes. Desta forma, os autores usaram também quatro modelos para o ajuste de equações hipsométricas.

Crechi et al. (1992), estudando a relação hipsométrica do pinheiro-do-paraná (Araucaria angustifolia) para intervalos de idade de 6 a 18 anos, ajustaram o modelo de Henriksen para as diferentes idades, independentes da densidade. Crechi (1996) selecionou o modelo de Curtis para ajustar alturas totais de (Araucaria angustifolia) em Missiones, na Argentina.

Este trabalho objetiva ajustar quatro modelos de relações hipsométricas (Trorey, Henricksen, Stoffels e Curtis) nos ecossistemas de mopane e selecionar o que melhor se ajusta aos dados de acordo com o coeficiente de determinação e erro padrão da estimativa. $\mathrm{O}$ ajuste das equações será validado e será realizada a análise de resíduos.

Os ecossistemas analisados localizam-se na província de Gaza, ao norte do Distrito de Mabalane, Posto Administrativo de Combomune, entre as coordenadas $23^{\circ} 10^{\prime} \mathrm{Sul}, 32^{\circ} 26,5^{\prime}$ Leste e $23^{\circ} 18^{\prime}$ Sul, $32^{\circ} 40^{\prime}$ Oeste.

A precipitação anual varia entre $400 \mathrm{~mm}$ e 600 mm e está concentrada entre os meses de novembro e fevereiro (Instituto Nacional de Investigação Agrária, 1999). A precipitação média anual é de $493 \mathrm{~mm}$, a evapotranspiração potencial (ETP) é de $1.485 \mathrm{~mm}$, a temperatura média anual é de $22,9{ }^{\circ} \mathrm{C}$ e a umidade relativa média anual é de 65\% (Instituto Nacional de Investigação Agrária, 1999).

A vegetação do distrito de Mabalane possui como espécies predominantes Colophospemum mopane
(Xanatsi) e a Androstachis johansonii (Cimbirre). Também sâo encontradas Terminalia sericea (Nkonola), Afzelia quanzenzis (Chanfuta), Strychnos madagascarensis (Nkwakwa), Vangueria infauta (Mphilua), Adansonia digitata (Embondeiro), entre outras (Instituto Nacional de Investigação Agrária, 1999).

\section{Levantamento de dados e modelos ajustados}

Foram usados dados de 425 árvores, obtidos em três parcelas permanentes de tamanho de $50 \mathrm{~m}$ x $50 \mathrm{~m}$ estabelecidas nos ecossistemas de Mopane em Mabalane, Província de Gaza, das quais, 400 foram usadas para finalidades de construção dos modelos de regressão e 25 foram separadas para a validação dos modelos. Nas áreas demarcadas foram medidas: altura em metros, com hipsómetro, e perímetro à altura do peito (PAP) em centímetros, com fita métrica. Estas medições foram feitas para todas as árvores com diâmetro a 1,30 m do solo (DAP) igual ou superiora $10 \mathrm{~cm}$. Para cada árvore foram estabelecidas coordenadas x e y que facilitarão a localização do mesmo indivíduo nas medições subsequentes.

Foram testados os modelos de Trorey, Henriksen, Stofells e Curtis (Tabela 1).

Tabela 1. Modelos hipsométricos ajustados para Colophospermum mopane.

\begin{tabular}{lc}
\hline \multicolumn{1}{c}{ Modelos } & \multicolumn{1}{c}{ Forma geral } \\
\hline Trorey & $H=\hat{a}_{0}+\hat{a}_{1} \times \mathrm{d}+\hat{\mathrm{a}}_{2} \mathrm{xd}^{2}$ \\
Henricksen & $H=\hat{a}_{0}+\hat{a}_{1} \times \ln \mathrm{d}$ \\
Stoffels & $\ln \mathrm{H}=\hat{a}_{0}+\hat{a}_{I} \times \operatorname{lnd}$ \\
Curtis & $H=\hat{a}_{0}+\hat{a}_{1} \times(1 / \mathrm{d})$ \\
\hline
\end{tabular}

$\mathrm{H}=$ altura em metros; $\beta_{0}, \beta_{1}$ e $\beta_{2}=$ Coeficientes do modelo: $\mathrm{d}=\mathrm{DAP}$.

\section{Seleção do melhor modelo}

O objetivo final de testar vários modelos de regressão é obter um modelo que apresente condições de explicar o fenômeno estudado, com baixa possibilidade de erro. Para essa escolha utilizam-se diversos critérios concomitantes como o coeficiente de determinação $\mathrm{R}_{\text {aj; }}^{2}$; erro padrão da estimativa $\mathrm{S}_{\mathrm{yx}}$; análise dos resíduos. 


\section{Coeficiente de determinação}

O Coeficiente de determinação expressa à quantidade de variação da variável dependente em função das variáveis independentes. Quanto mais próximo de um (1) melhor é o ajuste da equação.

$$
\begin{aligned}
& R^{2} \text { a j }=1-\left(\frac{n-1}{n-p} x \frac{\text { SQres }}{\text { SQtotal }}\right) \\
& \text { SQres }=\sum_{i=1}^{n}(Y i-\hat{Y}) \\
& \text { Stotal }=\sum_{i=1}^{n}(\mathrm{Yi}-\bar{Y})^{2}
\end{aligned}
$$

Onde: $\mathrm{R}^{2}$ aj= coeficiente de determinação ajustado; $\mathrm{n}=$ número de dados; $\mathrm{p}=$ número de coeficientes de cada modelo utilizado; SQres = soma dos quadrados dos resíduos; SQtotal = soma dos quadrados do total; $\mathrm{Yi}=$ alturas dominantes observados $(\mathrm{m}) ; \hat{Y} i=$ alturas dominantes estimados $(\mathrm{m}) ; \bar{Y}=$ média aritmética das alturas dominantes $(\mathrm{m})$;

\section{Erro padrão da estimativa}

$\mathrm{O}$ erro padrão da estimativa $\left(\mathrm{S}_{\mathrm{yx}}\right)$ mede a dispersão média entre os valores observados e os valores estimados ao longo da regressão. Quanto mais baixo for o valor de $\mathrm{S}_{\mathrm{yx}}$, melhor será a precisão das estimativas.

$$
S_{\mathrm{YX}}=\frac{\sqrt{\frac{\sum_{i=1}^{n}\left(Y_{i}-\hat{Y}_{i}\right)^{2}}{n-p}}}{\bar{Y}} \times 100
$$

Onde: $\mathrm{S}_{\mathrm{yx}} \%=$ erro padrão da estimativa em porcentagem; $\mathrm{p}=$ número de coeficientes do modelo; $\bar{Y}=$ média aritmética da alturas $(\mathrm{m}) ; \hat{Y} i=$ altura (m) estimada pelo modelo; Y $\mathrm{i}=$ altura observada (m).

Para os modelos cuja variável dependente sofreu transformação (logarítmica), os seus valores estimados são sujeitos ao efeito de discrepância logarítmica. Antes do recálculo do erro padrão da estimativa deve-se corrigir esta distorção multiplicando a altura estimada de cada árvore por um fator de correção de Meyer para obtenção do $S_{y x}$ recalculado.

Fator de Meyer $=\mathrm{e}^{0,5 *(\text { Syx }) 2}$ $\left(\mathrm{m}^{3}\right)$.

Onde: $e=2,718281828 ; S_{y x}=$ erro padrão da estimativa

\section{Validação dos modelos}

Comparou-se as alturas estimadas com as suas respectivas alturas reais, pelo teste de qui-quadrado $\left(\div^{2}\right.$ ) com nível de probabilidade de $95 \%$ :

$$
\dot{\div}^{2}=\sum \frac{(\mathrm{Fo}-\mathrm{Fest})^{2}}{\text { Fest }}
$$

Onde: $\dot{\div}^{2}=$ Qui-quadrado; Fo $=$ valor observado (real); Fest $=$ valor estimado.

Se o valor calculado de $\div^{2}$ for menor que seu valor tabelado ao nível de $95 \%$ de probabilidade, indicará que não existem diferenças significativas entre as alturas reais e estimadas, sendo a equação válida para a área de estudo.

\section{Análise gráfica dos resíduos}

A análise gráfica de resíduos é muito importante para avaliar se a estimativa é boa ou não ao longo da linha de regressão. Esta análise tem como objetivo verificar se o modelo selecionado pelo critério estatístico apresenta alguma tendência na estimativa da variável dependente.

$$
\mathrm{E} \mathrm{i}=\left(\frac{\mathrm{Yi}-\hat{Y} i}{\mathrm{Yi}}\right) \times 100
$$

Onde: $\mathrm{E}_{\mathrm{i}}=$ resíduo da i-ésima observação; $\mathrm{Y} \mathrm{i}=$ altura observada (m); $\hat{Y} i=$ altura estimada (m).

Este trabalho consistiu em cinco fases, nomeadamente ajuste de modelos hipsométricos; determinação das estatísticas de ajuste; validação do ajuste das equações; análise dos resíduos e a seleção do melhor modelo.

Os dados em altura possuem uma amplitude de 12,30 $\mathrm{m}$, com o valor mínimo de $2,7 \mathrm{~m}$ e o valor máximo de $15,0 \mathrm{~m}$, com o coeficiente de varição de $25,85 \%$ Em relação aos dados em diâmetro, a amplitude observada foi de $168 \mathrm{~cm}$, com o diâmetro mínimo de $16 \mathrm{~cm} \mathrm{e}$ diâmetro máximo de $184 \mathrm{~cm}$ e coeficiente de variação de 41,47\% (Tabela 2). Os coeficientes de regressão e as estatísticas de ajuste estão apresentados na Tabela 3.

Tabela 2. Apresentação das estatísticas dos dados.

\begin{tabular}{lcc}
\hline \multicolumn{1}{c}{ Medidas estatísticas } & Altura (m) & DAP (cm) \\
\hline Mínimo & 2,70 & 16,00 \\
Máximo & 15,00 & 184,00 \\
Média & 7,30 & 47,05 \\
Amplitude & 12,30 & 168,00 \\
Desvio padrão & 1,89 & 19,51 \\
Variância & 3,56 & 380,60 \\
CV\% & 25,83 & 41,47 \\
\hline
\end{tabular}


Tabela 3. Coeficientes de regressão e seus parâmetros estatísticos.

\begin{tabular}{lrrrrr}
\hline \multirow{2}{*}{ Modelos } & \multicolumn{3}{c}{ Coeficientes } & $\mathbf{S}_{\mathbf{y x}}$ & \multirow{2}{*}{$\mathbf{R}^{2}{ }_{\text {aj }}$} \\
\cline { 2 - 4 } corrigido & $\boldsymbol{\beta}_{\mathbf{0}}$ & \multicolumn{1}{c}{$\boldsymbol{\beta}_{\mathbf{1}}$} & $\boldsymbol{\beta}_{\mathbf{2}}$ & \\
\hline Trorey & 3,4389 & 0,0921 & $-0,00017$ & 0,1818 & 0,4932 \\
Henriksen & $-5,2637$ & 3,3365 & - & 0,1824 & 0,4884 \\
Stoffels & 0,1526 & 0,4782 & - & 0,1797 & 0,4992 \\
Curtis & 10,065 & 110,040 & - & 0,1934 & 0,4265 \\
\hline
\end{tabular}

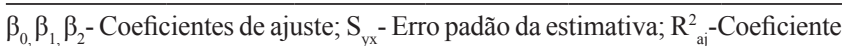
de determinação ajustado.

Considerando apenas as estatísticas de ajustes, o modelo de Stoffels foi o melhor em relação aos outros modelos, pois apresenta valor $\mathrm{R}_{\text {aj. }}^{2}$ de 0,4992 , mais alto em relação aos outros modelos e erro padrão da estimativa de 0,1797 menor que os outros modelos. Resultados do coeficiente de determinação, próximos a estes, foram encontrados por Barros et al. (2002), Batista et al. (2001). Segundo Machado et al. (1994), a relação hipsométrica não é uma relação dendrométrica muito forte devido a grande variabilidade de alturas para a mesma classe de diâmetro, resultando em estatísticas de ajustes muito baixos com valores não desejados em análise de regressão.
A validação do ajuste das equações foi determinada a partir da aplicação do teste de Qui-quadrado.

A análise gráfica de resíduos mostra que não há tendenciosidade em toda linha de regressão para todos os modelos testados (Figura 1).

$\mathrm{Na}$ Figura 2 estão apresentados os gráficos com os dados originais de alturas em função dos diâmetros e as curvas de pontos ajustadas para cada modelo de relação hipsométrica. O modelo de Curtis mostrou-se inadequado para representar os dados originais, pois apresenta uma curva com tendência assitótica a partir de diâmetros acima de $60 \mathrm{~cm}$. Contrariamente, os modelos de Trorey, Soffels e Henricksen mostraram-se adequados para representar os dados observados de altura em função de diâmetro, considerando que estes modelos mostraram-se muito semelhantes entre si e bastante próximos à tendência dos dados.

O melhor modelo após análise dos parâmetros estatísticos e análise gráfica dos resíduos foi o modelo de Stofels. Todos os modelos analisados são válidos para a região e os desvios não apresentam tentenciosidade ao longo da linha de regressão.

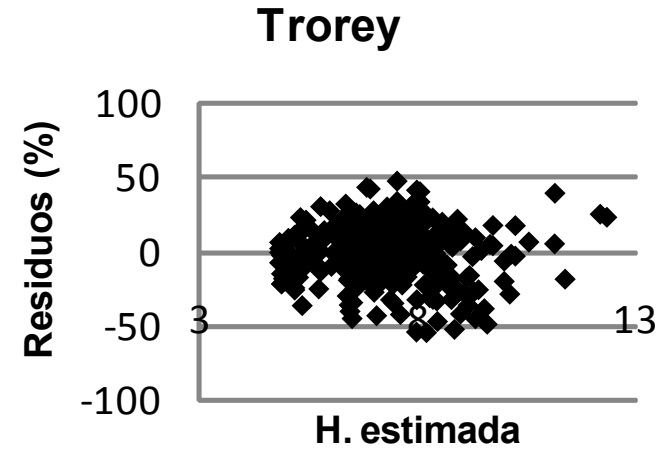

Henricksen

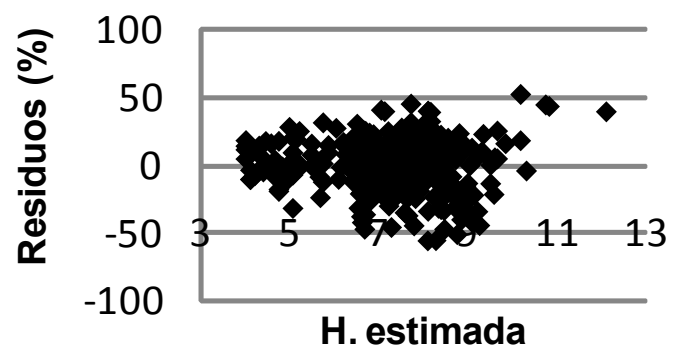

Stoffels

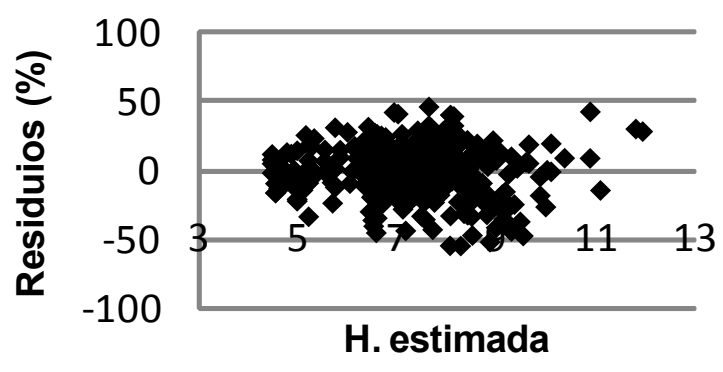

Curtis

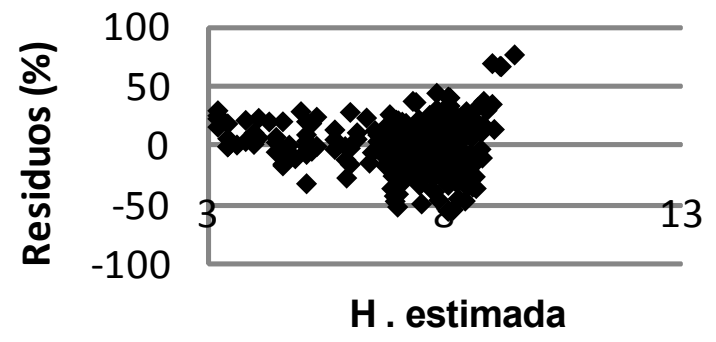

Figura 1. Relações hipsométricas de Colophopermum mopane, na Pronvíncia de Gaza, Moçambique. 

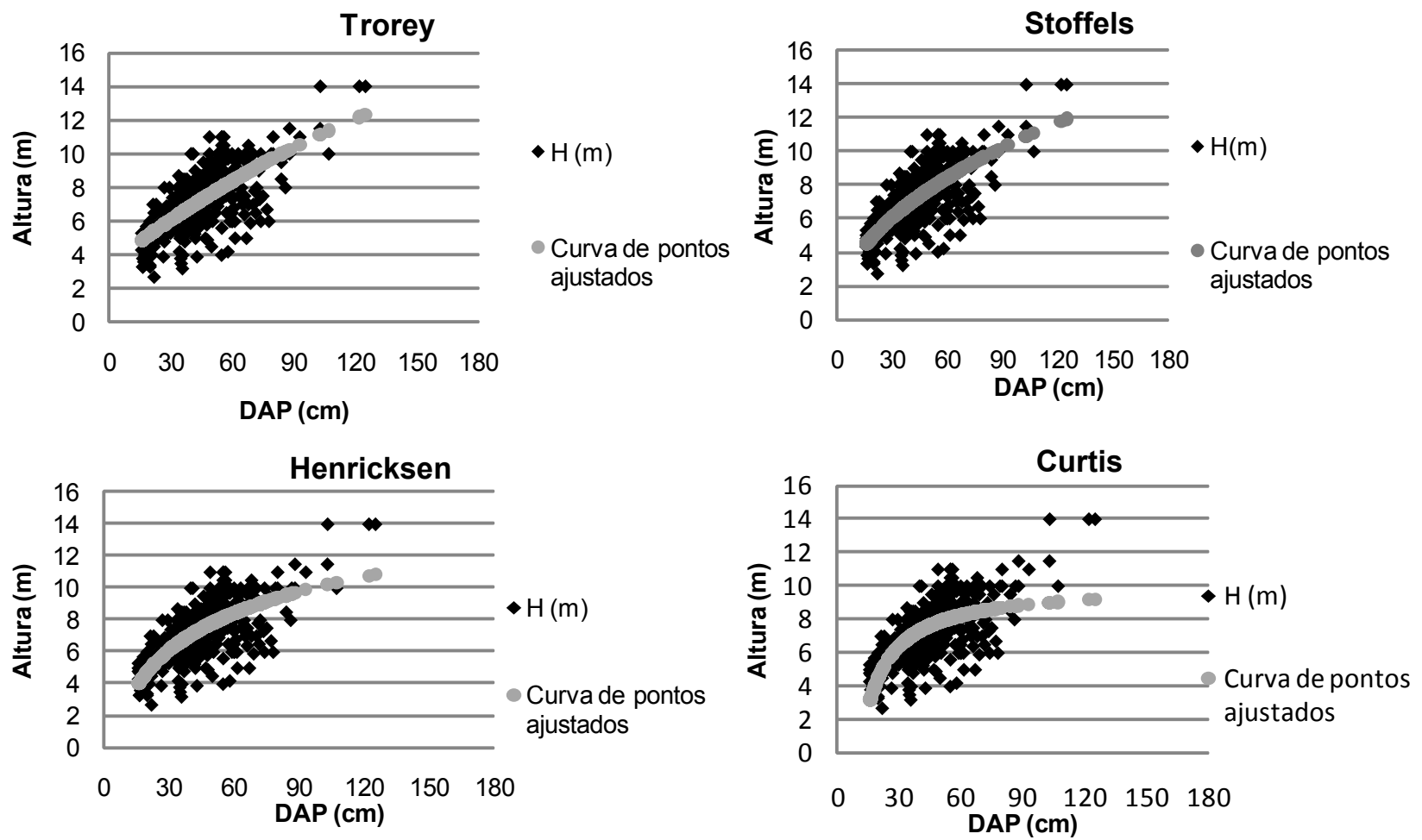

Figura 2. Dispersão de alturas observadas e curva de pontos ajustados.

\section{Agradecimentos}

$\mathrm{O}$ autor agradece aos colegas do setor de Ecologia e Manejo Florestal, do Centro de Investigação Florestal do Instituto de Investigação Agrária de Moçambique, principalmente ao técnico Nazaré Mabjaia, pelo apoio e ajuda na coleta de dados. Agradece também ao engenheiro. Esperança Chamba, pela revisão do trabalho.

\section{Referências}

BARROS, D.; MACHADO, S.; ACERBÍ, F. W.; SCOLFORO, J. R. Comportamento de modelos hipsométricos tradicionais e genéricos para plantações de Pinus ocarpa em diferentes tratamentos. Boletim de Pesquisa Florestal, Colombo, n. 45 p. 3-28, jul./dez. 2002.

BATISTA J.; COUTO H.; MARQUESINI M.; Desempenho de modelos de relações hipsométricas em três tipos de floresta nomeadamente floresta nativa. Scientia Forestalis, n. 60, p. 149$163,2001$.
CRECHI, E. H.; FRIEDL, R. A.; FERNÁNDEZ, R. A. La relación hipsométrica através del tiempo en Araucaria angustifolia (Bert). O. Ktze. Yvyraretá, Eldorado, n 3, p. 86-92, 1992.

CRECHI, E. H Efeito da densidade de plantações sobre a produção, Cresciemnto e sobrevivência de Araucaria angustifolia (Bert). O. Ktze. em Missiones, Argentina. 1996. 171 f. Dissertação (Mestrado em Engenharia Florestal) - Setor de Ciências Agrárias, Universidade Federal do Paraná, Curitiba.

MACHADO, S.; BAILEY, R.; BASSO, S.; BEVILACQUA, V. Análise do comportamento da relação hipsométrica com respeito à idade para plantações de Pinus elliotti no Estado do Paraná. Cerne, v. 1, n. 1, p. 5-12, 1994.

INSTITUTO NACIONAL DE INVESTIGAÇÃO AGRÁRIA. Inventário dos Recursos de Terra na Comunidade de Gerés, Distrito de Mabalane. Maputo, 1999. p. 77 p. (Série terra e água, 1).

TOMÉ, M.; RIBEIRO, F.; Relações Hipsométricas geral para Eucalyptus globulus Labill em Portugal. Silva Lusitana, Lisboa, v. 15 , n. 1, p. 41-55, 2007.

VEIGA, R.; CARVALHO, C.; BRASIL, M.; Equações hipsométricas e de volume para Eucalytus alba Reino. Revista Floresta, Botucatu, v. 5, n. 1 p. 64-78, 1974. 
